\title{
Stationary nonlinear Schrödinger equation on the graph for the triangle with outgoing bonds
}

\author{
K. K. Sabirov ${ }^{1,2}$, M. Aripov ${ }^{3}$, D. B. Sagdullayev ${ }^{4}$ \\ ${ }^{1}$ Turin Polytechnic University in Tashkent, 17 Niyazov Str. 100095 Tashkent, Uzbekistan \\ ${ }^{2}$ Tashkent University of Information Technologies, Amir Temur Str. 108, Tashkent, Uzbekistan \\ ${ }^{3}$ Department of Mathematics, National University of Uzbekistan, \\ University Str. 1, 100174, Tashkent, Uzbekistan \\ ${ }^{4}$ Department of High Matnematics, Tashkent State University of Economy, \\ Uzbekistan Str., 49, 100003, Tashkent, Uzbekistan \\ karimjonsabirov80@gmail.com
}

PACS 05.45.Yv

DOI 10.17586/2220-8054-2017-8-1-24-28

\begin{abstract}
We consider the stationary (cubic) nonlinear Schrödinger equation (NLSE) on a simple metric graph in the form of a triangle with three infinite outgoing bonds. Exact solutions are obtained for primary star graph with the boundary vertex conditions providing the wave function weights continuity and flux conservation for the case of repulsive nonlinearity.
\end{abstract}

Keywords: Nonlinear Schrödinger equation, a metric graph, repulsive nonlinearity.

Received: 10 July 2016

Revised: 25 August 2016

\section{Introduction}

The nonlinear evolution equation on metric graphs have attracted much attention over the last decade [1-10]. Such interest is caused by the possibility of modeling nonlinear waves and soliton transport in networks and branched structures by nonlinear wave equations on metric graphs. The latter is of practical importance for the problem of engineering network architectures providing tunable transport of heat, spin, charge, light and information transport in networks. In addition, such equations can be used for to model soliton transport in DNA double helix, conducting polymers, branched optical waveguides, Josephson junction networks and Bose-Einstein condensation in branched traps.

Earlier, the problem of soliton transport in branched structures and networks was mainly studied within the tight-binding approach [11]. However, such an approach doesn't provide comprehensive treatment of the problem, thus one needs to use continuous NLSE on graphs.

Considerable progress has been made so far in the obtaining of soliton solutions of different nonlinear wave equations on metric graphs. The integrability of nonlinear Schrödinger equation on simple metric graphs was shown and soliton solutions providing reflectionless transmission of solitons through the graph vertex have been obtained in [1]. Exact solutions for the stationary NLSE and their stability were studied in the Refs. [3-7]. Dispersion relations for the linear and NLSE on networks are discussed in [12]. Sine-Gordon equation on metric graphs has been studied in $[8,9]$. The NLSE and soliton transport in planar graphs were studied in [10].

In this paper, we treat the stationary NLSE on the graph for a triangle with outgoing bonds shown in Fig. 1. Graphs are the systems consisting of bonds which are connected at the vertices [13]. The bonds are connected according to a rule that is called the topology of a graph. The topology of a graph is given in terms of so-called adjacency matrix (or connectivity matrix) which can be written as $[14,15]$ :

$$
C_{i j}=C_{j i}= \begin{cases}1 & \text { if } i \text { and } j \text { are connected, } \quad i, j=1,2, \ldots, V . \\ 0 & \text { otherwise, }\end{cases}
$$

This paper is organized as follows. In the next section we will present formulation of the problem for the graph shown in Fig. 1. Section III presents derivation of the solution for the stationary NLSE on this graph by considering the repulsive nonlinearity. In section IV, we discuss the extension of these results to the case of the attractive nonlinearity. Finally, section V presents some concluding remarks. 


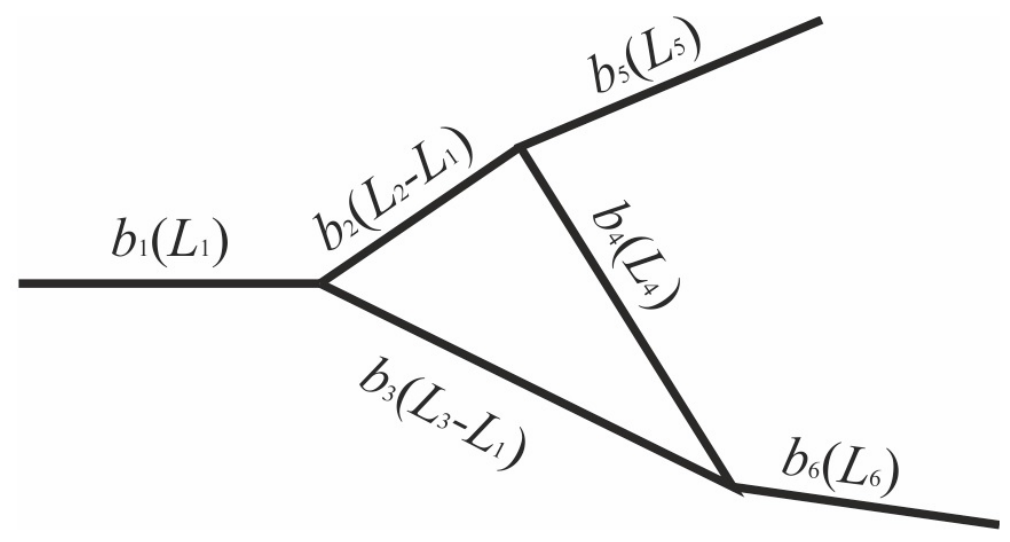

FIG. 1. The graph for the triangle with outgoing bonds

\section{Stationary Schrödinger equation on the graph for the triangle with outgoing infinite bonds}

Consider a metric graph presented in Fig. 1 which consists of a triangle with finite sides connected to outgoing infinite leads $\left(b_{1,4,5,6} \sim\left(0 ; L_{1,4,5,6}\right), b_{2,3} \sim\left(L_{1} ; L_{2,3}\right)\right)$.

Then, the time-independent (stationary) NLSE on each bond of $b$ of such graph can be written as:

$$
-\psi_{b}^{\prime \prime}+\beta_{b}\left|\psi_{b}\right|^{2} \psi_{b}=\lambda^{2} \psi_{b}, \quad \beta_{b}>0
$$

Eq. (1) is a multi-component equation in which the components are connected to each other through the vertex boundary conditions providing the "glue" for the bonds at the branching of points of a graph. For the graph in Fig. 1, such boundary conditions can be written as:

$$
\begin{array}{r}
\left.\psi_{1}(x)\right|_{x=0}=0,\left.\psi_{5}(x)\right|_{x=L_{5}}=0,\left.\psi_{6}(x)\right|_{x=L_{6}}=0 \\
\left.\sqrt{\beta_{1}} \psi_{1}(x)\right|_{x=L_{1}}=\left.\sqrt{\beta_{2}} \psi_{2}(x)\right|_{x=L_{1}}=\left.\sqrt{\beta_{3}} \psi_{3}(x)\right|_{x=L_{1}}, \\
{\left.\left[\frac{1}{\sqrt{\beta_{1}}} \frac{\partial \psi_{1}(x)}{\partial x}-\frac{1}{\sqrt{\beta_{2}}} \frac{\partial \psi_{2}(x)}{\partial x}-\frac{1}{\sqrt{\beta_{3}}} \frac{\partial \psi_{3}(x)}{\partial x}\right]\right|_{x=L_{1}}=0} \\
\left.\sqrt{\beta_{2}} \psi_{2}(x)\right|_{x=L_{2}}=\left.\sqrt{\beta_{4}} \psi_{4}(x)\right|_{x=0}=\left.\sqrt{\beta_{5}} \psi_{5}(x)\right|_{x=0} \\
\left.\frac{1}{\sqrt{\beta_{2}}} \frac{\partial \psi_{2}(x)}{\partial x}\right|_{x=L_{2}}-\left.\frac{1}{\sqrt{\beta_{4}}} \frac{\partial \psi_{4}(x)}{\partial x}\right|_{x=0}-\left.\frac{1}{\sqrt{\beta_{5}}} \frac{\partial \psi_{5}(x)}{\partial x}\right|_{x=0}=0 \\
\frac{1}{\left.\sqrt{\beta_{3}} \psi_{3}(x)\right|_{x=L_{3}}}=\left.\sqrt{\beta_{4}} \psi_{4}(x)\right|_{x=L_{4}}=\left.\sqrt{\beta_{6}} \psi_{6}(x)\right|_{x=0} \\
\left.\frac{\partial \psi_{3}(x)}{\partial x}\right|_{x=L_{3}}-\left.\frac{1}{\sqrt{\beta_{4}}} \frac{\partial \psi_{4}(x)}{\partial x}\right|_{x=L_{4}}-\left.\frac{1}{\sqrt{\beta_{6}}} \frac{\partial \psi_{6}(x)}{\partial x}\right|_{x=0}=0
\end{array}
$$

and the wave function is normalized as:

$$
\sum_{j=1}^{6} \int_{b_{j}}\left|\psi_{j}(x)\right|^{2} d x=1 .
$$

Dirichlet boundary conditions are imposed at the bond edges.

\section{Solution of the time-independent NLSE on the graph for the triangle with outgoing bonds}

The solution of Eq. (1) can be written in the form:

$$
\psi_{b}(x)=f_{b}(x) e^{i \gamma_{b}},
$$

where $b$ is bond index, $\gamma_{b}=$ const, $f_{b}(x)$ is a real function obeying the equation:

$$
-f_{b}^{\prime \prime}+\beta_{b} f_{b}^{3}=\lambda^{2} f_{j}
$$

From the boundary conditions we have $\gamma_{b}=\gamma$ and it is clear that the functions $f_{b}$ should fulfill Eqs. (2) - (9). 
Exact solutions for Eq. (11) for finite interval and periodic boundary conditions can be found in the Refs. [18, 19]. Here, we consider this problem for the metric graph boundary conditions given by Eq. (2). The solution of Eq. (11) fulfilling these boundary conditions can be written as:

$$
f_{b}(x)=B_{b} \operatorname{sn}\left(\alpha_{b} x+\delta_{b} \mid k_{b}\right)
$$

where $\operatorname{sn}(a x \mid k)$ are the Jacobian elliptic functions [20], $\delta_{1}=0, \delta_{5,6}=-\alpha_{5,6} L_{5,6}$.

Inserting the last equation into Eq. (10) and comparing the coefficients of similar terms we have:

$$
B_{b}=\sigma_{b} \sqrt{\frac{2}{\beta_{b}}} \alpha_{b} k_{b}, \quad \lambda^{2}=\alpha_{b}^{2}\left(1+k_{b}^{2}\right),
$$

where $\sigma_{b}= \pm 1$.

Using Eqs. (2)-(9) and the relations [20]:

$$
\begin{gathered}
\int_{a}^{b} \operatorname{sn}^{2}(\alpha(x-c) \mid k) d x=\frac{1}{k^{2}} \int_{a}^{b}\left[1-\operatorname{dn}^{2}(\alpha(x-c) \mid k)\right] d x \\
=\frac{1}{k^{2}}(b-a)-\frac{1}{\alpha k^{2}} E[\operatorname{am}(\alpha(b-c)) \mid k]+\frac{1}{\alpha k^{2}} E[\operatorname{am}(\alpha(a-c)) \mid k],
\end{gathered}
$$

we obtain the following system of transcendental equations with respect to $\alpha_{b}, \delta_{b}$ and $k_{b}$ :

$$
\begin{aligned}
& \sqrt{\beta_{1}} B_{1} \operatorname{sn}\left(\alpha_{1} L_{1} \mid k_{1}\right)=\sqrt{\beta_{2}} B_{2} \operatorname{sn}\left(\alpha_{2} L_{1}+\delta_{2} \mid k_{2}\right)=\sqrt{\beta_{3}} B_{3} \operatorname{sn}\left(\alpha_{3} L_{1}+\delta_{3} \mid k_{3}\right), \\
& \left.\left.\frac{B_{1} \alpha_{1}}{\sqrt{\beta_{1}}} \operatorname{cn}\left(\alpha_{1} L_{1} \mid k_{1}\right) \operatorname{dn}\left(\alpha_{1} L_{1} \mid k_{1}\right)-\frac{B_{2} \alpha_{2}}{\sqrt{\beta_{2}}} \operatorname{cn}\left(\alpha_{2} L_{1}+\delta_{2}\right) \mid k_{2}\right) \operatorname{dn}\left(\alpha_{2} L_{1}+\delta_{2}\right) \mid k_{2}\right) \\
& -\frac{B_{3} \alpha_{3}}{\sqrt{\beta_{3}}} \operatorname{cn}\left(\alpha_{3} L_{1}+\delta_{3} \mid k_{3}\right) \operatorname{dn}\left(\alpha_{3} L_{1}+\delta_{3} \mid k_{3}\right)=0, \\
& \sqrt{\beta_{2}} B_{2} \operatorname{sn}\left(\alpha_{2} L_{2}+\delta_{2} \mid k_{2}\right)=\sqrt{\beta_{4}} B_{4} \operatorname{sn}\left(\delta_{4} \mid k_{4}\right)=\sqrt{\beta_{5}} B_{5} \operatorname{sn}\left(\delta_{5} \mid k_{5}\right), \\
& \left.\left.\frac{B_{2} \alpha_{2}}{\sqrt{\beta_{2}}} \operatorname{cn}\left(\alpha_{2} L_{2}+\delta_{2} \mid k_{2}\right) \operatorname{dn}\left(\alpha_{2} L_{2}+\delta_{2} \mid k_{2}\right)-\frac{B_{4} \alpha_{4}}{\sqrt{\beta_{4}}} \operatorname{cn}\left(\delta_{4}\right) \mid k_{4}\right) \operatorname{dn}\left(\delta_{4}\right) \mid k_{4}\right) \\
& -\frac{B_{5} \alpha_{5}}{\sqrt{\beta_{5}}} \operatorname{cn}\left(\delta_{5} \mid k_{5}\right) \operatorname{dn}\left(\delta_{5} \mid k_{5}\right)=0 \\
& \sqrt{\beta_{3}} B_{3} \operatorname{sn}\left(\alpha_{3} L_{3}+\delta_{3} \mid k_{3}\right)=\sqrt{\beta_{4}} B_{4} \operatorname{sn}\left(\alpha_{4} L_{4}+\delta_{4} \mid k_{4}\right)=\sqrt{\beta_{6}} B_{6} \operatorname{sn}\left(\delta_{6} \mid k_{6}\right), \\
& \frac{B_{3} \alpha_{3}}{\sqrt{\beta_{3}}} \operatorname{cn}\left(\alpha_{3} L_{3}+\delta_{3} \mid k_{3}\right) \operatorname{dn}\left(\alpha_{3} L_{3}+\delta_{3} \mid k_{3}\right)-\frac{B_{4} \alpha_{4}}{\sqrt{\beta_{4}}} \operatorname{cn}\left(\alpha_{4} L_{4}+\delta_{4} \mid k_{4}\right) \operatorname{dn}\left(\alpha_{4} L_{4}+\delta_{4} \mid k_{4}\right) \\
& -\frac{B_{6} \alpha_{6}}{\sqrt{\beta_{6}}} \operatorname{cn}\left(\delta_{6} \mid k_{6}\right) \operatorname{dn}\left(\delta_{6} \mid k_{6}\right)=0 \text {, } \\
& \frac{B_{1}^{2}}{k_{1}^{2}} L_{1}+\frac{B_{2}^{2}}{k_{2}^{2}}\left(L_{2}-L_{1}\right)+\frac{B_{3}^{2}}{k_{3}^{2}}\left(L_{3}-L_{1}\right)+\frac{B_{4}^{2}}{k_{4}^{2}} L_{4}+\frac{B_{5}^{2}}{k_{5}^{2}} L_{5}+\frac{B_{6}^{2}}{k_{6}^{2}} L_{6} \\
& +\frac{B_{2}^{2}}{k_{2}^{2} \alpha_{2}} E\left[\operatorname{am}\left(\alpha_{2} L_{1}+\delta_{2} \mid k_{2}\right) \mid k_{2}\right]+\frac{B_{3}^{2}}{k_{3}^{2} \alpha_{3}} E\left[\operatorname{am}\left(\alpha_{3} L_{1}+\delta_{3} \mid k_{3}\right) \mid k_{3}\right]+\sum_{j=4}^{6} \frac{B_{j}^{2}}{k_{j}^{2} \alpha_{j}} E\left[\operatorname{am}\left(\delta_{j} \mid k_{j}\right) \mid k_{j}\right] \\
& =1+\sum_{j=1}^{6} \frac{B_{j}^{2}}{k_{j}^{2} \alpha_{j}} E\left[\operatorname{am}\left(\alpha_{j} L_{j}+\delta_{j} \mid k_{j}\right) \mid k_{j}\right],
\end{aligned}
$$

where $\delta_{1}=0, \delta_{5,6}=-\alpha_{5,6} L_{5,6}, E(\varphi \mid k)$ and $\operatorname{am}(u \mid k)$ are the incomplete elliptic integral of the second kind and the Jacobi amplitude, respectively. In the general case, this system can be solved using the different (e.g., Newton's or Krylov's method) iteration schemes. However, below, we will show the solvability of this system for two special cases.

First case. Let:

$$
\frac{4 n_{1}+1}{L_{1}}=\frac{4\left(n_{2,3}^{(2)}-n_{2,3}^{(1)}\right)}{L_{2,3}-L_{1}}=\frac{4 n_{4}}{L_{1}}=\frac{4 n_{5,6}+1}{L_{5,6}},
$$


where $n_{j} \in \mathbf{N} \cup\{0\}, j=1,2,3,4,5,6, n_{2,3}^{(2)}>n_{2,3}^{(1)}$. Choosing:

$$
\begin{gathered}
\alpha_{1,5,6}=\frac{4 n_{1,5,6}+1}{L_{1,5,6}} K\left(k_{1,5,6}\right), \alpha_{2,3}=\frac{4\left(n_{2,3}^{(2)}-n_{2,3}^{(1)}\right)}{L_{2,3}-L_{1}} K\left(k_{2,3}\right), \alpha_{4}=\frac{4 n_{4}}{L_{4}} K\left(k_{4}\right), \\
\delta_{2,3}=\left(\frac{4\left(n_{2,3}^{(2)}-n_{2,3}^{(1)}\right)}{L_{2,3}-L_{1}}+1\right) K\left(k_{2,3}\right), \delta_{4}=K\left(k_{4}\right)
\end{gathered}
$$

we have:

$$
\alpha_{j}=\alpha, \quad k_{j}=k, j=1,2,3,4,5,6, \quad \sigma_{1,2,3,4}=-\sigma_{5,6} .
$$

Here, $K(k)$ is the complete elliptic integrals of the first kind.

Using Eq. (19) and the relations:

$$
\begin{aligned}
& \operatorname{am}(u+2 K(k) \mid k)=\pi+\operatorname{am}(u \mid k), \\
& E(n \pi \pm \varphi \mid k)=2 n E(k) \pm E(\varphi \mid k),
\end{aligned}
$$

we have:

$$
g(k) \equiv 2 \frac{\left(4 n_{1}+1\right)^{2}}{L_{1}^{2}}\left(\frac{L_{1}}{\beta_{1}}+\frac{L_{2}-L_{1}}{\beta_{2}}+\frac{L_{3}-L_{1}}{\beta_{3}}+\frac{L_{4}}{\beta_{4}}+\frac{L_{5}}{\beta_{5}}+\frac{L_{6}}{\beta_{6}}\right) K(k)(K(k)-E(k))-1=0 .
$$

The solvability of Eq. (21) is equivalent to that of NLSE on a primary star graph. Therefore we will prove the solvability of this equation. Since the following relations are valid:

$$
\lim _{k \rightarrow 0} g(k)=-1, \lim _{k \rightarrow 1} g(k)=+\infty,
$$

and $g(k)$ is a continuous function of $k$ on the interval $(0 ; 1)$ it follows that Eq. (21) has a root.

Second case. Now we consider another special case given by the relations:

$$
\begin{gathered}
\alpha_{1}=\frac{(-1)^{n_{1}} p_{1}+2 n_{1} K\left(k_{1}\right)}{L_{1}}, \alpha_{2,3}=\frac{(-1)^{n_{2,3}^{(2)}} p_{2,3}-(-1)^{n_{2,3}^{(1)}} p_{1}+2\left(n_{2,3}^{(2)}-n_{2,3}^{(1)}\right) K\left(k_{2,3}\right)}{L_{2,3}-L_{1}}, \\
\alpha_{4}=\frac{\left.(-1)^{n_{4}^{(1)}} p_{3}-(-1)^{n_{4}^{(2)}} p_{2}\right)+2 n_{4}^{(1)} K\left(k_{4}\right)}{L_{4}}, \\
\delta_{2,3}=\frac{(-1)^{n_{2,3}^{(1)}} p_{1} L_{2,3}-(-1)^{n_{2,3}^{(2)}} p_{2,3} L_{1}+2\left(n_{2,3}^{(1)} L_{2,3}-n_{2,3}^{(2)} L_{1}\right) K\left(k_{2,3}\right)}{L_{2,3}-L_{1}}, \delta_{4}=(-1)^{n_{4}^{(2)}} p_{2}+2 n_{4}^{(2)} K\left(k_{4}\right),
\end{gathered}
$$

where $-K\left(k_{j}\right) \leq p_{m} \leq K\left(k_{j}\right), m=1,2,3, j=1,2,3,4,5,6, n_{1,4,5,6}, n_{2,3}^{(1),(2)} \in \mathbf{N}, n_{1}, n_{2}^{(1)}, n_{3}^{(1)}$ or $n_{2}^{(2)}, n_{4}^{(2)}$, $n_{5}$ or $n_{3}^{(2)}, n_{4}^{(1)}, n_{6}$ cannot be odd or even at the same time and show existence of the solution of the system given by Eqs. (13)-(19). From Eqs. (13), (15) and (17) we obtain

$$
\alpha_{b}=\alpha, k_{b}=k, \sigma_{1,2,3,4}=-\sigma_{5,6} .
$$

From Eqs. (14), (16) and (18) we have:

$$
\frac{(-1)^{n_{1}}}{\beta_{1}}=\frac{(-1)^{n_{2}^{(1)}}}{\beta_{2}}+\frac{(-1)^{n_{3}^{(1)}}}{\beta_{3}}, \quad \frac{(-1)^{n_{2}^{(2)}}}{\beta_{2}}=\frac{(-1)^{n_{4}^{(1)}}}{\beta_{4}}+\frac{(-1)^{n_{5}}}{\beta_{5}}, \quad \frac{(-1)^{n_{3}^{(2)}}}{\beta_{3}}=\frac{(-1)^{n_{4}^{(2)}}}{\beta_{4}}+\frac{(-1)^{n_{6}}}{\beta_{6}} .
$$

Furthermore, it follows from the last equation and Eq. (19) that:

$$
\begin{array}{r}
g(k) \equiv 4\left(\frac{(-1)^{n_{1}} p+2 n_{1} K\left(k_{1}\right)}{L_{1}}\right)\left(\frac{n_{1}}{\beta_{1}}+\frac{n_{2}^{(2)}-n_{2}^{(1)}}{\beta_{2}}+\frac{n_{3}^{(2)}-n_{3}^{(1)}}{\beta_{3}}+\frac{n_{4}^{(1)}}{\beta_{4}}+\frac{n_{5}}{\beta_{5}}+\frac{n_{6}}{\beta_{6}}\right) \times \\
\times(K(k)-E(k))-1=0 .
\end{array}
$$

We have:

$$
\lim _{k \rightarrow 0} g(k)=-1, \quad \lim _{k \rightarrow 1} g(k)=+\infty .
$$

Since $g(k)$ is a continuous function of $\mathrm{k}$ on the interval $(0 ; 1)$, it follows from the last relations that Eq. (22) has a root.

Thus, the stationary NLSE on a metric graph presented in Fig. 1 can be solved with the same success as that for finite interval, considered in the Refs. [18, 19,21]. 


\section{Conclusion}

In this paper, we have studied the stationary nonlinear Schrödinger equation with cubic nonlinearity on the graph having the form of a triangle connected to finite outgoing leads considering the case of attractive nonlinearity. Explicit analytic solutions were obtained by imposing vertex boundary conditions providing continuity of the wave function weights and flux conservation. The method can also be extended for the case of repulsive nonlinearity, too.

The above results can be useful for modeling of different branched systems, where the static solitons appear, e.g. for Bose-Einstein condensate in networks [24-27] charge and energy modeling in DNA double helix [28-30] and branched optical waveguides [31,32], on In principle, the method developed in this work can be extended for more complicated topologies having multiple junctions.

\section{Acknowledgements}

This work is partially supported by the grant of the Committee for the Coordination Science and Technology Development.

\section{References}

[1] Sobirov Z., Matrasulov D., Sabirov K., Sawada S., and Nakamura K. Integrable nonlinear Schrödinger equation on simple networks: Connection formula at vertices, Phys. Rev. E, 2010, 81, P. 066602.

[2] Nakamura K., Sobirov Z.A., Matrasulov D.U., and Sawada S. Transport in simple networks described by an integrable discrete nonlinear Schrödinger equation. Phys. Rev. E, 2011, 84, P. 026609.

[3] Adami R., Cacciapuoti C., Finco D., and Noja D. FAST SOLITONS ON STAR GRAPHS. Rev. Math. Phys., 2011, 23, P. 409.

[4] Adami R., Cacciapuoti C., Finco D., and Noja D. Stationary states of NLS on star graphs. Europhys. Lett., 2012,100, P. 10003.

[5] Adami R., Noja D., and Ortoleva C. Orbital and asymptotic stability for standing waves of a nonlinear Schrdinger equation with concentrated nonlinearity in dimension three. J. Math. Phys., 2013, 54, P. 013501.

[6] Sabirov K.K., Sobirov Z.A., Babajanov D., and Matrasulov D.U. Stationary nonlinear Schrödinger equation on simplest graphs. Phys. Lett. A, 2013, 377, P. 860 .

[7] Noja D. Nonlinear Schrödinger equation on graphs: recent results and open problems. Philos. Trans. R. Soc. A, 2014, 372, P. 20130002

[8] Susanto G.H. and van Gils S.A. Existence and stability analysis of solitary waves in a tricrystal junction. Phys. Lett. A, 2005, 338, P. 239.

[9] Caputo J.-G., Dutykh D. Nonlinear waves in networks: Model reduction for the sine-Gordon equation. Phys. Rev. E, 2014 , 90, P. 022912.

[10] Uecker H., Grieser D., Sobirov Z., Babajanov D., and Matrasulov D. Soliton transport in tubular networks: transmission at vertices in the shrinking limit. Phys. Rev. E, 2015, 91, P. 023209.

[11] Burioni R., Cassi D., Sodano P., Trombettoni A. and Vezzani A. Soliton Propagation in Chains with Simple non Local Defects. Physica D, 2006, 216, P. 71 .

[12] Banica V.,Ignat L. Dispersion for the Schrödinger Equation on Networks, Arxiv: 1103.0429.

[13] Harary F. Graph Theory. Addison-Wesley, Reading, 1969.

[14] Tsampikos Kottos and Uzy Smilansky. Periodic orbit theory and spectral statistics for quantum graphs. Ann.Phys., 1999, 76, P. 274.

[15] Sven Gnutzmann and Uzy Smilansky. Quantum Graphs: Applications to Quantum Chaos and Universal Spectral Statistics. Adv.Phys., 2006, 55, P. 527.

[16] Gnutzmann S., Smilansky U., Derevyanko S. Stationary scattering from a nonlinear network. Phys. Rev. A, 2011, 83, P. 033831.

[17] Cascaval R.C., Hunter C.T. Linear and nonlinear Schrödinger equations on simlest networks. Libertas Math., 2010,30 , P. 85.

[18] Carr L.D., Charles W. Clark and Reinhardt W.P. Stationary solutions of the one-dimensional nonlinear Schrdinger equation. I. Case of repulsive nonlinearity, Phys. Rev. A, 2000, 62, P. 063610.

[19] Carr L.D., Charles W. Clark and Reinhardt W.P. Stationary solutions of the one-dimensional nonlinear Schrdinger equation. II. Case of attractive nonlinearity, Phys. Rev. A, 2000, 62, P. 063611.

[20] Bowman F. Introduction to Elliptic Functions, with Applications. Dover, New York, 1961.

[21] Carr L.D., Mahmud K.W. and Reinhardt W.P. Tunable tunneling: An application of stationary states of Bose-Einstein condensates in traps of finite depth. Phys. Rev. A, 2001, 64, P. 033603.

[22] D'Agosta R., Malomed B.A. and Presilla C. Stationary solutions of the GrossPitaevskii equation with linear counterpart. Phys. Lett. A, 2000, 275, P. 424.

[23] Infeld E.,Zin P.,Gocalek J. and Trippenbach M. Statics and dynamics of BEC in double square well potentials. Phys. Rev. E, 2006, 74, P. 026610.

[24] Bianconi G., Barabasi A-L. Bose-Einstein Condensation in Complex Networks. Phys.Rev.Lett. A, 2001, 86, P. 5632.

[25] Vidal E.J.G.G., Lima R.P.A., and Lyra M.L. Bose-Einstein condensation in the infinitely ramified star and wheel graphs. Phys. Rev. E, 2011, 83, P. 061137.

[26] De Oliveira I.N., dos Santos T.B., de Moura F.A.B.F., Lyra M.L. and Serva M. Critical behavior of the ideal-gas Bose-Einstein condensation in the Apollonian network. Phys. Rev. E, 2013, 88, P. 022139.

[27] Castellanos E., Chacon-Acosta G. Polymer BoseEinstein condensates. Phys.Lett. B, 2013, 722, P. 119.

[28] Yakushevich L.V. Nonlinear Physics of DNA. Wiley, Chichester, 2004.

[29] Peyrard M. Nonlinearity, 2004, 17, P. R1.

[30] Zdravkovica S., Sataric M.V. Nonlinear Schrdinger equation and DNA dynamics. Phys.Lett. A, 2008, 373, P. 126.

[31] Leo G., Assanto G. Multiple branching of vectorial spatial solitary waves in quadratic media. Opt. Commun., 1998,146, P. 356.

[32] Santos M.C. Dynamic Y-branched structures in quadratic nonlinear media. Opt. Commun., 2000, 180, P. 167. 